\title{
Mitral leaflet anatomy revisited
}

\author{
Jason L. Quill, BS, ${ }^{\mathrm{a}}$ Alexander J. Hill, PhD, ${ }^{\mathrm{b}}$ Timothy G. Laske, PhD, ${ }^{\mathrm{b}}$ Ottavio Alfieri, MD, ${ }^{\mathrm{c}}$ and Paul A. Iaizzo, PhD ${ }^{\mathrm{a}}$
}

Objective: The aims of this work were to employ functional imaging capabilities of the Visible Heart laboratory and endoscopic visualization of mitral valves in perfusion-fixed specimens to better characterize variability in mitral valve leaflet anatomy and to provide a method to classify mitral leaflets that varies from the current nomenclature.

Methods: We gathered functional endoscopic video footage (11 isolated reanimated human hearts) and static endoscopic anatomical images (38 perfusion-fixed specimens) of mitral leaflets. Commissure and cleft locations were charted using Carpentier's accepted description.

Results: All hearts had 2 commissures separating anterior and posterior leaflets. "Standard" clefts separating P1/ $\mathrm{P} 2$ were found in $66 \%$ of hearts $(\mathrm{n}=25)$, and standard clefts separating $\mathrm{P} 2 / \mathrm{P} 3$ were present in $71 \%$ of hearts $(\mathrm{n}=$ 27). "Deviant" clefts occurred in each region of the anterior leaflet (A1, A2, A3), and their relative occurrences were $5 \%, 8 \%$, and $13 \%(\mathrm{n}=2,3,5)$, respectively. Deviant clefts were found in posterior leaflets: $13.2 \%$ in P1 $(\mathrm{n}=5), 32 \%$ in $\mathrm{P} 2(\mathrm{n}=12)$, and $21 \%$ in $\mathrm{P} 3(\mathrm{n}=8)$.

Conclusions: Humans elicit complex and highly variable mitral valve anatomy. We suggest a complementary, yet simple nomenclature to address variation in mitral valve anatomy by describing clefts as either standard or deviant and locating regions in which they occur (A1 to A3 or P1 to P3).

The human mitral valve is a very complex, dynamic, and highly variable structure. Current nomenclature describes it as a bileaflet valve with chordae tendinae connecting the leaflets to 2 ventricular papillary muscles; this definition was made popular by Carpentier and colleagues. ${ }^{1,2}$ Furthermore, the leaflet nomenclature typically describes an anterior and posterior leaflet, each divided by 2 commissures. The posterior leaflet is then further divided into P1, P2, and $\mathrm{P} 3$ scallop regions by clefts. The anterior leaflet descriptions do not include the labeling of any clefts, but the leaflet is subdivided into the $\mathrm{A} 1, \mathrm{~A} 2$, and $\mathrm{A} 3$ regions that oppose the scallops of the posterior leaflet (Figure 1). Because the term commissure is defined as "a line at which two things are joined," we have chosen to reserve the term commissure to describe where the anterior leaflet joins the posterior leaflet, and we will use the term cleft for a division between scallops on either the anterior or posterior leaflet.

Several previous investigators have described the mitral valve leaflet anatomy, and each has added unique insights. For example, in 1970, Ranganathan and colleagues ${ }^{3}$ classi- $^{-}$ fied the mitral leaflet based on its functional zones. They

\footnotetext{
From the Departments of Surgery and Biomedical Engineering, University of Minnesota, ${ }^{\mathrm{a}}$ Minneapolis, Minn; Medtronic, Inc, ${ }^{\mathrm{b}}$ Minneapolis, Minn; and San Raffaele Hospital of Milan, ${ }^{\mathrm{c}}$ Milan, Italy.

This work was supported in part by the Institute for Engineering in Medicine at the University of Minnesota and by Medtronic, Inc.

Received for publication May 15, 2008; revisions received Aug 25, 2008; accepted for publication Oct 8, 2008

Address for reprints: Paul A. Iaizzo, PhD, Department of Surgery, University of Minnesota, B172 Mayo, MMC 107, 420 Delaware Street SE, Minneapolis, MN 55455 (E-mail: iaizz001@umn.edu).

J Thorac Cardiovasc Surg 2009;137:1077-81

0022-5223/\$36.00

Copyright (c) 2009 by The American Association for Thoracic Surgery

doi:10.1016/j.jtcvs.2008.10.008
}

described an "edge zone," a "clear zone," and a "basal zone" based upon the thickness and location of these leaflet tissues. This extensive study (46 specimens) was highly descriptive, but the subsequently developed nomenclatures and/or definitions were mainly utilized by anatomists and not clinicians. Furthermore, valve scallops were classified based upon the presence of "cleft" chordae, in which $92 \%$ of the posterior leaflets observed contained 3 scallops. ${ }^{4}$ Likewise, Chiechi and associates ${ }^{5}$ classified "accessory leaflets" on the posterior cusp. Yet, neither group charted the exact locations of clefts (defined as slits that separate the scallops of the leaflets) if and when they occurred. The simplicity and practicality of Carpentier's anatomic description led to its widespread use after being introduced in 1976. It should be noted that Kumar and colleagues ${ }^{6}$ went on to classify the leaflets of the mitral valve using a numbering scheme for the scallops; however, Carpentier's description remains the most commonly used.

Importantly, from a purely anatomic perspective, use of the terms anterior and posterior leaflets has been disputed. Specifically, common anatomic terms used to describe the human heart are typically based upon examination of a specimen in the so-called "valentine" position, with the heart oriented such that the interventricular septum is parallel to the long axis of the body, with the apex positioned inferiorly. This position is not attitudinally correct, that is, it does not describe how the heart is positioned in the intact thoracic cavity. In other words, terms such as anterior and posterior are thus applied to the incorrect surfaces of the heart and, thereby, to structures within the heart including the mitral valve. Therefore, if one employs an attitudinally correct nomenclature, the terms anterior and posterior leaflets become imprecise. Alternative names have been proposed based on 


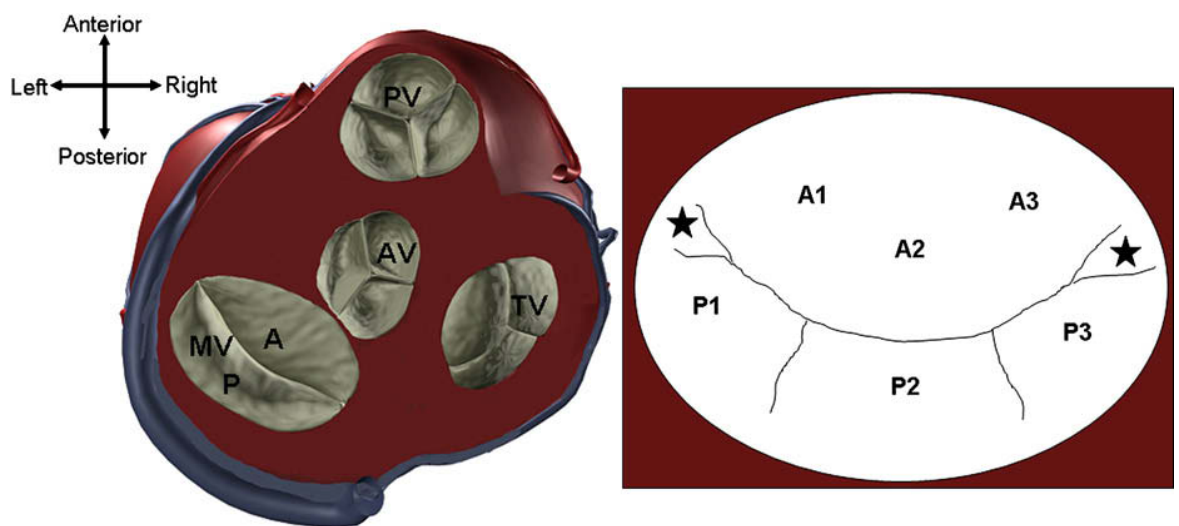

FIGURE 1. The relative orientation of the human mitral valve $(M V)$ with respect to the other cardiac valves is shown on the left. This is a view of the base of the heart looking toward the apex, with the atria and great vessels removed. The anterior $(A)$ and posterior $(P)$ leaflets of the mitral valve were labeled along with the aortic valve $(A V)$, the pulmonary valve $(P V)$, and the tricuspid valve $(T V)$. The alpha-numeric diagram on the right shows Carpentier's description ${ }^{1,2}$ of the mitral valve leaflets. Kumar et al's description ${ }^{6}$ differs only by the addition of the starred regions and nomenclature.

the correct position of the heart- the aortic and mural leaflets, respectively. ${ }^{7}$ The authors feel it is important to acknowledge this discrepancy in common nomenclature but will use the Carpentier nomenclature for this study. Rather than attempting to provide a new nomenclature that perhaps more accurately and precisely describes the attitudinally correct anatomy of the mitral leaflets, a large variation in the number of scallops and their location is acknowledged. We simply propose a manner to describe this variation in anatomy relative to the widely employed Carpentier definition.

Our aims were to employ both the functional imaging capabilities of the Visible Heart laboratory and the endoscopic visualization of mitral valves in perfusion-fixed specimens to better characterize variability in mitral valve leaflet anatomy and to provide a method to classify mitral leaflet variations that differs from the current nomenclature.

\section{METHODS}

The protocol and procedures employed for this research were reviewed and approved by the Human Subjects Committee Internal Review Board at the University of Minnesota. Specimens were procured through the Anatomy Bequest Program at the University of Minnesota or from LifeSource, Inc (St Paul, MN).

Functional endoscopic video footage was gathered from 11 isolated reanimated human hearts using previously described Visible Heart methodologies. ${ }^{8}$ Briefly, the hearts from organ donors that were deemed not viable for transplantation were administered a cardioplegic solution and cooled. Upon arrival in the lab and after cannulation, the hearts were subsequently perfused with a Krebs-Henseleit buffer and warmed to normal body temperature $\left(37^{\circ} \mathrm{C}\right)$. Subsequently, these hearts were defibrillated and all hearts elicited a normal, noninnervated sinus rhythm. The utilization of the clear buffer solution allowed for functional, intracardiac endoscopic imaging within the beating heart, including the functioning mitral valve from above and below. ${ }^{8}$

In addition, static endoscopic anatomic images of the mitral leaflets were gathered from 38 perfusion-fixed specimens, including the 11 aforementioned hearts using Visible Heart methodologies. These hearts were obtained fresh with their great vessels intact, cannulated, and subsequently fixed by pressurizing the heart $(40$ to $50 \mathrm{~mm} \mathrm{Hg}$ ) with a $10 \%$ formalin so- lution, during which time the heart remained submerged in a formalin-filled tank. All hearts were fixed using this method for greater than 48 hours. Following perfusion fixation, and while keeping the specimen fully intact, the leaflets of the mitral valve were observed from an endoscope placed into the left atrium and navigated through a pulmonary vein ostium.

Next, the nomenclature proposed by Carpentier was used to diagram the locations of each cleft within the anterior and posterior leaflets. If a cleft was found in either of the 2 locations described by Carpentier, it was referred to as a "standard" cleft. Any clefts that were found in regions described as being scalloped regions by the current nomenclature were termed "deviant" clefts. In this way, clefts that were uniquely located beyond the classic mitral valve description were tabulated in the leaflet region in which the deviant cleft occurred.

\section{RESULTS}

The locations of commissures and clefts relative to Carpentier's description for the 38 analyzed hearts are shown in Figure 2. All hearts analyzed had 2 commissures separating the anterior and posterior leaflets. Carpentier's description describes 2 clefts in the posterior leaflet (termed standard clefts). The standard cleft that separates P1 from P2 (ie, from Carpentier's description of 2 clefts in the posterior leaflet) was found to be present at the described locations in $66 \%$ of the hearts $(n=25$ of 38$)$, and the standard cleft that separates P2 from P3 was present in $71 \%$ of the hearts $(\mathrm{n}=27)$.

All other clefts were defined to be located at places that deviated from Carpentier's description (ie, within regions described as having scallops). Deviant clefts occurred in the anterior leaflet in each region $(\mathrm{A} 1, \mathrm{~A} 2$, and $\mathrm{A} 3)$ and their relative occurrences were $5 \%, 8 \%$, and $13 \%(\mathrm{n}=$ 2,3 , and 5), respectively. Such deviant clefts also were found in the posterior leaflets and were more frequently observed than deviant clefts in the anterior leaflet. The occurrence of deviant clefts per region is as follows: $13.2 \%$ in $\mathrm{P} 1(\mathrm{n}=5), 32 \%$ in $\mathrm{P} 2(\mathrm{n}=12)$, and $21 \%$ in $\mathrm{P} 3(\mathrm{n}=$ 8). Interestingly, 1 mitral valve had a deviant cleft in 


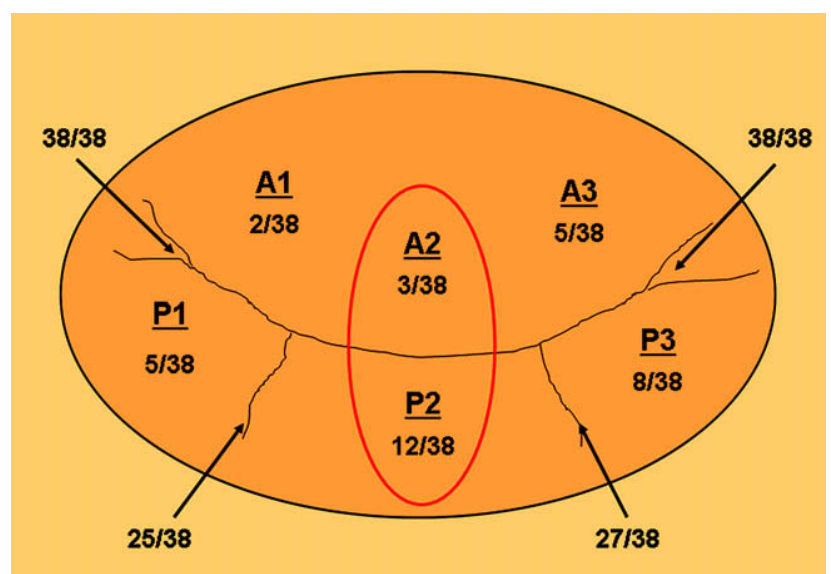

FIGURE 2. Of the 38 human hearts studied, cleft locations were compared with Carpentier's description as shown by the diagram and labeled regions. Clefts that occurred within a scallop region instead of the diagrammed commissure positions were tallied in the scallop region they were discovered and termed "deviant" clefts.

both the $\mathrm{A} 2$ and $\mathrm{P} 2$ regions. It was also noted that none of the hearts analyzed had more than 1 deviant cleft in any single leaflet region.

The average number of anterior leaflet scallops was $1.2 \pm$ 0.4 , and the average number of posterior leaflet scallops was $3.0 \pm 1.0$. The relative variability of the scallops associated with each of the 38 hearts is presented as a histogram in Figure 3.

Figure 4 shows functional images of 2 human mitral valves during systole (left panel) and diastole (right). The mitral valve in the top panes of the figure has 2 anterior scallops and 5 posterior scallops, with deviant clefts in the A3, $\mathrm{P} 1$, and $\mathrm{P} 2$ regions. The mitral valve in the lower panels of the figure has deviant clefts in the $\mathrm{A} 1, \mathrm{P} 1, \mathrm{P} 2$, and $\mathrm{P} 3$ regions and lacks a standard cleft between P1 and P2.

\section{DISCUSSION}

Our observations are consistent with the notion that the human mitral valve is a very complex, dynamic, and highly variable structure. In every region of the anterior and posterior leaflets, deviant clefts were observed in at least 1 heart, yet they were present less often than standard clefts. In our specimens, the anterior leaflets were found to have fewer deviant clefts than the posterior leaflets, and deviant clefts appeared most often in the P2 regions. It should also be noted that standard clefts were found in the majority of the hearts analyzed but were not always present at the described position.

Here we also provided a simple and practical way for describing mitral valve leaflet anatomy that differs from the common nomenclature. Our review of the literature identified that the most common configuration for leaflet anatomy was the description provided by Carpentier, who describes 3 pos-

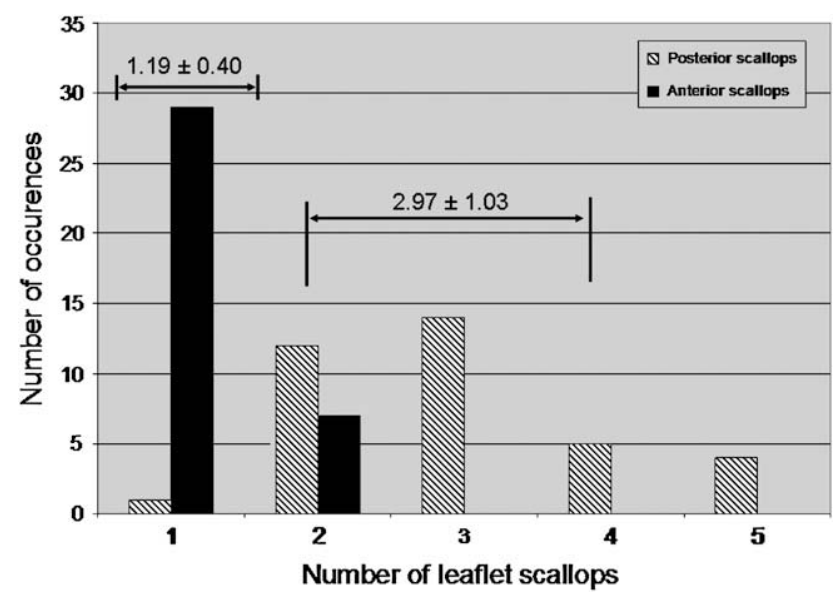

FIGURE 3. Shown is a histogram of anterior and posterior scallop frequencies, which indicates that the average number of anterior leaflet scallops is $1.19 \pm 0.40$ and the number of posterior leaflet scallops is $2.97 \pm 1.03$.

terior leaflet scallops with 2 clefts separating them, 2 commissures separating the anterior and posterior leaflet, and 1 anterior scallop. We believe that our study supports the use of this common nomenclature but provides a novel extension to accurately describe variations in mitral leaflet clefts.

Functional anatomic studies, such as video analyzed from the reanimated human hearts in the Visible Heart laboratory, can provide new insights that are difficult to observe in conventional anatomy studies. Anatomic studies of the mitral leaflets are typically analyzed with the valve in the systolic position, as this is how surgeons see the valve. However, Figure 4 shows 2 examples of hearts that were analyzed using functional images. Clefts that were not readily apparent in the systolic position become obvious in the early diastolic period. Basing anatomic studies on the systolic position of the mitral valve may soon change with the improvement of noninvasive imaging technologies.

An accurate assessment of in vivo mitral valve anatomy will become even more important in the future, as more and more interventional procedures are developed and performed to repair or replace dysfunctional valves. More specifically, detailed anatomic analyses on the presence or absence of leaflet clefts would be performed prior to or during a repair procedure. Currently, real-time 3-dimensional echocardiography has the capability to visualize mitral leaflets at the level of the mitral annulus, with enough resolution to observe individual leaflet scallops. ${ }^{9,10}$ This imaging technique may potentially provide evidence of deviant clefts described in this study prior to or during a procedure. It has been suggested that 3-dimensional echocardiography will play an important role in percutaneous mitral valve repair, ${ }^{11}$ an arena in which the authors believe deviant clefts could affect device delivery.

Mitral valve dysfunction can be related to several factors, including diseased leaflets, ${ }^{12}$ annular changes, ${ }^{13}$ abnormal 

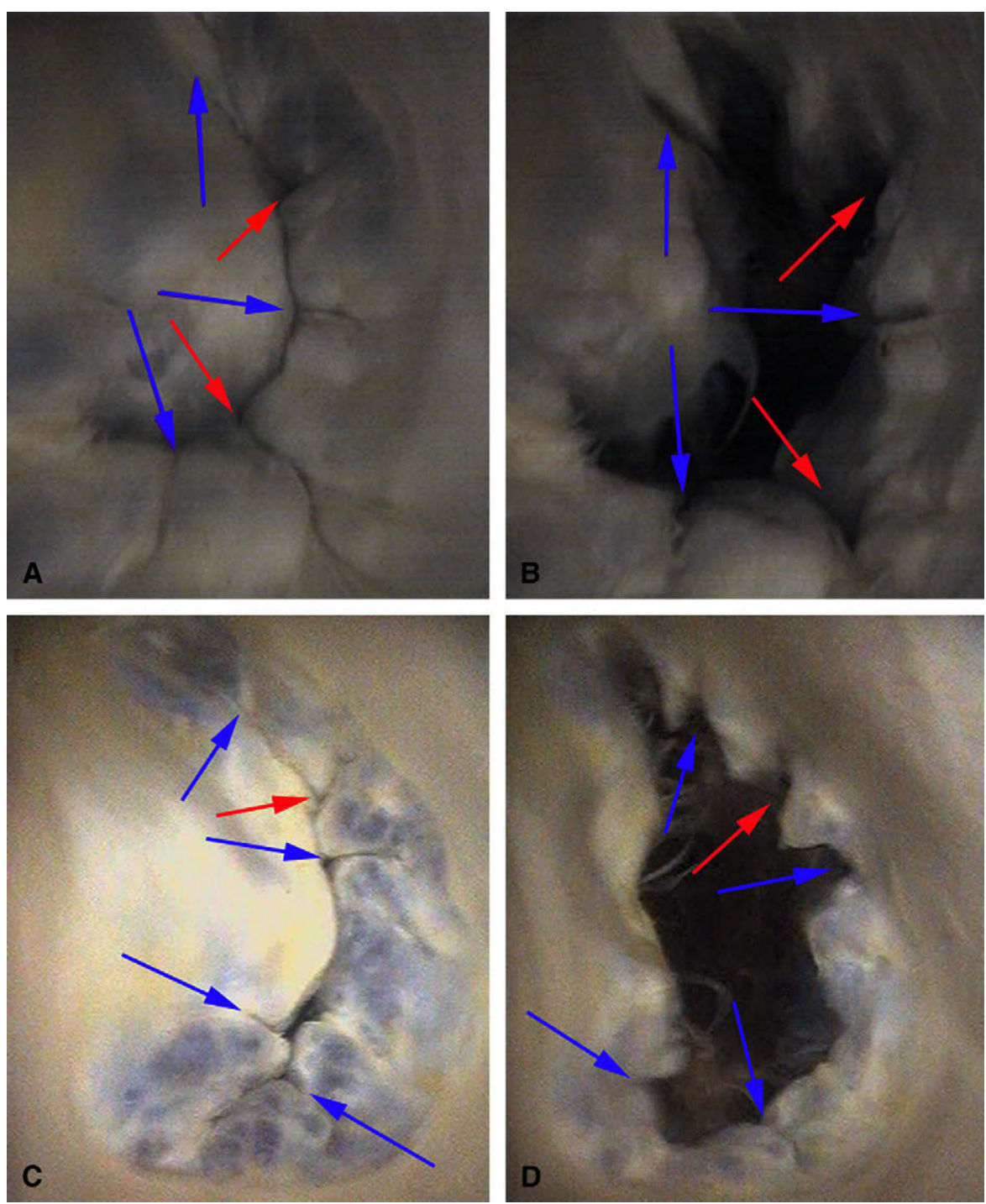

FIGURE 4. Two mitral valves are shown in systolic $(A, C)$ and diastolic $(B, D)$ positions with the anterior leaflet to the left and the posterior leaflet to the right. Blue arrows signify deviant clefts, and red arrows signify standard clefts. The mitral valve in panels $A$ and $B$ contains 3 deviant clefts in regions P1, P2, and A3, with 2 standard clefts. The mitral valve in panels $C$ and $D$ has 4 deviant clefts in the A1, P1, P2, and P3 regions. This mitral valve has 1 standard cleft between $\mathrm{P} 2$ and $\mathrm{P} 3$ but is lacking a standard cleft between P1 and P2.

or damaged chordae, ${ }^{14}$ and ventricular dilatation ${ }^{15}$ causing displacement of the papillary muscles. Although surgical repair of the mitral valve includes several mature and proven techniques, many patients remain untreated ${ }^{16}(58,000$ surgical mitral procedures in the United States in 2005 versus a prevalence of 2.3 million individuals with moderate to severe mitral regurgitation). This unmet clinical need has inspired the recent development of numerous new devices for transcatheter repair of the mitral valve. These transcatheter devices can be subdivided into 5 general types: (1) devices for Alfieri-type edge-to-edge repair; (2) indirect annuloplasty devices deployed into the coronary sinus; (3) direct annuloplasty devices placed on or near the mitral annulus; (4) devices for dimensional control of the left ventricle or left atrium; and (5) devices for mitral valve replacement. ${ }^{17,18}$

Relative to our study, the approach to mitral valve repair of particular interest is the edge-to-edge technique, in which a stitch is placed to join the anterior and posterior leaflets at the location of regurgitation. ${ }^{19-21}$ This technique is most commonly used in patients with A2 or P2 prolapse, and the simplicity of the edge-to-edge technique has led to opportunities for percutaneous valve repair. $^{22-24}$ The authors theorize that the A2 or P2 deviant clefts demonstrated in this study could affect the deployment of transcatheter edge-to-edge devices in select patients and that routine use of 3-dimensional 
echocardiography could identify these clefts and aid in procedural success.

\section{CONCLUSIONS}

Humans elicit complex and highly variable mitral valve anatomy. We expanded the description of these variations in leaflet anatomy in the context of currently accepted nomenclature. A useful way of describing variation in mitral valve anatomy is to describe the clefts as either standard or deviant and to locate the leaflet regions in which they occur (A1 to $\mathrm{A} 3$ or $\mathrm{P} 1$ to $\mathrm{P} 3$ ).

We thank the patients and their families for the generous donations of these heart specimens for this research and both the University of Minnesota Bequest Program and LifeSource for assistance with securing organs. We also thank Sara Anderson for her assistance with data collection, Gary Williams for preparation of the images, and Monica Mahre for her help with the manuscript submission.

\section{References}

1. Carpentier A. Cardiac valve surgery-the "French correction." J Thorac Cardiovasc Surg. 1983;86:323-37.

2. Carpentier A, Branchini B, Cour JC, Asfaou E, Villani M, Deloche A, et al. Congenital malformations of the mitral valve in children. Pathology and surgical treatment. J Thorac Cardiovasc Surg. 1976;72:854-66.

3. Ranganathan N, Lam JH, Wigle ED, Silver MD. Morphology of the human mitral valve. II. The value leaflets. Circulation. 1970;41:459-67.

4. Lam JH, Ranganathan N, Wigle ED, Silver MD. Morphology of the human mitral valve. I. Chordae tendineae: a new classification. Circulation. 1970; 41:449-58.

5. Chiechi M, Lees W, Thompson R. Functional anatomy of the normal mitral valve. J Thorac Surg. 1956;32:378.

6. Kumar N, Kumar M, Duran CM. A revised terminology for recording surgical findings of the mitral valve. J Heart Valve Dis. 1995;4:70-5; discussion 76-7.

7. Wilcox BR, Cook AC, Anderson RH, eds. Surgical anatomy of the heart. 3rd ed. New York: Cambridge University Press; 2004.

8. Hill AJ, Laske TG, Coles JA Jr, Sigg DC, Skadsberg ND, Vincent SA, et al. In vitro studies of human hearts. Ann Thorac Surg. 2005;79:168-77.
9. Garcia-Orta R, Moreno E, Vidal M, Ruiz-Lopez F, Oyonarte JM, Lara J, et al. Three-dimensional versus two-dimensional transesophageal echocardiography in mitral valve repair. J Am Soc Echocardiogr. 2007;20:4-12.

10. Sugeng L, Coon P, Weinert L, Jolly N, Lammertin G, Bednarz JE, et al. Use of real-time 3-dimensional transthoracic echocardiography in the evaluation of mitral valve disease. J Am Soc Echocardiogr. 2006;19:413-21.

11. Ryan LP, Salgo IS, Gorman RC, Gorman JH 3rd. The emerging role of threedimensional echocardiography in mitral valve repair. Semin Thorac Cardiovasc Surg. 2006;18:126-34.

12. Roberts WC. Morphologic features of the normal and abnormal mitral valve. Am J Cardiol. 1983;51:1005-28.

13. Yiu SF, Enriquez-Sarano M, Tribouilloy C, Seward JB, Tajik AJ. Determinants of the degree of functional mitral regurgitation in patients with systolic left ventricular dysfunction: a quantitative clinical study. Circulation. 2000; 102:1400-6.

14. Hickey AJ, Wilcken DE, Wright JS, Warren BA. Primary (spontaneous) chordal rupture: Relation to myxomatous valve disease and mitral valve prolapse. J Am Coll Cardiol. 1985;5:1341-6.

15. Kono T, Sabbah HN, Rosman H, Alam M, Jafri S, Goldstein S. Left ventricular shape is the primary determinant of functional mitral regurgitation in heart failure. J Am Coll Cardiol. 1992;20:1594-8.

16. Stuge O, Liddicoat J. Emerging opportunities for cardiac surgeons within structural heart disease. J Thorac Cardiovasc Surg. 2006;132:1258-61.

17. Babaliaros V, Block P. State of the art percutaneous intervention for the treatment of valvular heart disease: a review of the current technologies and ongoing research in the field of percutaneous valve replacement and repair. Cardiology. 2007; 107:87-96.

18. Feldman T, Leon MB. Prospects for percutaneous valve therapies. Circulation. 2007;116:2866-77.

19. Nakanishi K, Raman J, Hata M, Buxton B. Early outcome with the alfieri mitral valve repair. J Cardiol. 2001;37:263-6.

20. Alfieri O, Maisano F, De Bonis M, Stefano PL, Torracca L, Opizzi M, et al. The double-orifice technique in mitral valve repair: a simple solution for complex problems. J Thorac Cardiovasc Surg. 2001;122:674-81.

21. Alfieri O, Maisano F. An effective technique to correct anterior mitral leaflet prolapse. J Card Surg. 1999;14:468-70.

22. Alfieri O, Maisano F, Colombo A. Percutaneous mitral valve repair procedures Eur J Cardiothorac Surg. 2004;26:S36-7; discussion S37-8.

23. Alfieri O, De Bonis M, Lapenna E, Regesta T, Maisano F, Torracca L, et al "Edge-to-edge" repair for anterior mitral leaflet prolapse. Semin Thorac Cardiovasc Surg. 2004;16:182-7.

24. Alfieri O, Maisano F, Colombo A, Pappone C, La Canna G, Zangrillo A. Percutaneous mitral valve repair: an attractive perspective and an opportunity for teamwork. Ital Heart J. 2004;5:723-6. 